\title{
Bibliotecas universitarias y proyección social: diferencias y extremos en América Latina
}

\author{
César R. Nureña*
}

Artículo recibido:

21 de agosto de 2018

Artículo aceptado:

9 de enero de 2019

Artículo de investigación

\section{Resumen}

El análisis de los reglamentos de servicios de setenta bibliotecas universitarias latinoamericanas (de Perú, México, Brasil, Argentina, Colombia, Chile y Bolivia) revela que existen enormes diferencias en las maneras en que los bibliotecarios de esta parte del mundo entienden y llevan a la práctica nociones sobre el acceso al conocimiento y el papel de proyección social de las universidades. En algunos países, estas bibliotecas operan como servicios públicos para la comunidad (Brasil, México y Argentina), mientras que en otros se establecen múltiples restricciones a los usuarios externos (con situaciones extremas en Perú). El artículo discute las implicancias de estos hallazgos en relación

* Facultad de Ciencias Sociales, Universidad Nacional Mayor de San Marcos, Lima, Perú

cnurena@gmail.com

INVESTIGACIÓN BIBLIOTECOLÓGICA, vol. 33, núm. 80, julio/septiembre, 2019, México, ISSN: 2448-8321 pp. 117-132 
con los roles de las bibliotecas y la función universitaria de proyección social.

Palabras clave: Bibliotecas Universitarias; Usuarios Externos; Servicios Bibliotecarios; América Latina.

University libraries and social outreach: differences and extremes in Latin America César R. Nureña

\section{Abstract}

The analysis of the service regulations of 70 Latin American university libraries (from Peru, Mexico, Brazil, Argentina, Colombia, Chile and Bolivia) reveals enormous differences in how librarians in this part of the world understand and carry on practical notions about access to knowledge and the university's role of community service. In some countries, these libraries operate as public services for the community (Brazil, Mexico and Argentina), while in others there are multiple restrictions to external users (with Peru as an extreme case). The article discusses the implications of these findings in relation to the roles of libraries and the university function of social outreach.

Key Words: University Libraries; External Users; Library Services, Latin America.

\section{INTRODUCCIÓN}

T a enorme importancia que en nuestros días han cobrado la información Wy el conocimiento impone desafíos mayores a las bibliotecas y a los bibliotecarios, que se han ido adaptando a la nueva "sociedad del conocimiento", modernizando sus actividades y servicios (Mainka et al., 2013; Materska, 2004) y democratizando el acceso a la información (Stilwell, 2018; Martin, 1990). En este escenario, las bibliotecas académicas desempeñan funciones claves, pues operan en los entornos donde con mayor intensidad se utilizan, 
generan y transmiten los conocimientos. Así pues, en apariencia, habrían quedado atrás los tiempos en que estas bibliotecas funcionaban bajo modelos monacales, como celosas guardianas de saberes atesorados hasta con cadenas, a los que sólo pequeñas élites de privilegiados podían acceder. Actualmente, en cambio, en gran parte del mundo las bibliotecas universitarias se alinean decididamente con las funciones que por tradición se atribuyen a las universidades: la investigación, la formación profesional y la proyección social (Martin, 1990; Torres, 2005).

Este trabajo se enfoca en este último punto, el de la proyección social universitaria, en su relación con los roles de las bibliotecas académicas. Si las universidades sólo tienen razón de ser en la medida en que se deben a las sociedades que las albergan, entonces cobra pleno sentido el que sus bibliotecas no limiten sus servicios a las pequeñas comunidades universitarias, sino que los extiendan también a las poblaciones de los contextos en que se desenvuelven. De hecho, esto es lo que típicamente ocurre en los países que con mayor ventaja participan en la sociedad del conocimiento. En Europa y Norteamérica, pero también en buena parte de Asia y América Latina, las bibliotecas universitarias son, a la vez, bibliotecas públicas abiertas a todos los ciudadanos (Atkinson, 2018; Courtney, 2001; Russell et al., 1992; Weare y Stevenson, 2012).

Sin embargo, esta tendencia se da de manera desigual en el espacio latinoamericano, donde como se demuestra más adelante conviven dos grandes esquemas de doctrina y práctica bibliotecaria: por un lado, el enfoque que coloca a las bibliotecas universitarias como servicios públicos y punta de lanza de la proyección social universitaria (Herrera y Pérez, 2009; Torres, 2005) y, por otro lado, el modelo premoderno en que se las concibe como espacios exclusivos de élites socioeconómicas o ilustradas.

Partiendo de estas ideas, este trabajo tiene como propósito describir y analizar el panorama latinoamericano, en materia de acceso a servicios de información, por parte de la ciudadanía en las bibliotecas universitarias de la región. La pregunta que guía esta pesquisa es ¿qué tan abiertas o restrictivas son las bibliotecas universitarias latinoamericanas frente a la posibilidad de ofrecer servicios de información a los ciudadanos de sus sociedades, más allá de los públicos afiliados a las instituciones académicas en que funcionan?

Para responder a esta pregunta, se realizó un estudio basado en un análisis de los reglamentos de servicios de sendos conjuntos de bibliotecas universitarias de siete países de América Latina, análisis que permitió construir un instrumento destinado a cuantificar los grados de apertura o restricción frente a los usuarios "externos", que se definen en general como personas no afiliadas a las instituciones en que funcionan tales bibliotecas (Russell et al., 1992). Dicho 
instrumento contempla cinco categorías que se ordenan jerárquicamente, con el acceso irrestricto en un extremo y la completa exclusión en el otro, escala que sirvió también para asignar puntajes a los conjuntos de bibliotecas de cada país, con lo que se obtuvo un panorama de las tendencias predominantes y diferencias entre ellos.

Como complemento, se analiza más en profundidad el caso de Perú, donde se encontró una situación extrema de barreras que las bibliotecas imponen a los usuarios externos. El carácter límite de este caso nacional amerita una consideración de algunos factores socioculturales y políticos que podrían estar interviniendo en el fenómeno hallado, cuestión que es objeto de discusión en la sección correspondiente.

El trabajo concluye con algunos comentarios acerca de las diferencias que se presentan entre los países de América Latina en relación con el acceso a la información en sus bibliotecas universitarias, tema que tiene hondas implicancias en el campo de la biblioteconomía y en el terreno de las políticas universitarias.

\section{Métodos}

Se realizó un estudio exploratorio, cuantitativo y descriptivo, enfocado en los niveles de apertura o restricción que presentan las bibliotecas universitarias de siete países latinoamericanos respecto del acceso de usuarios externos a sus servicios. Para esto, se seleccionó una muestra propositiva y no probabilística de setenta bibliotecas "centrales" o núcleos de "sistemas de bibliotecas” de igual número de universidades de Perú, México, Colombia, Brasil, Chile, Argentina y Bolivia. Para la selección se tomó como referencia el QS World University Rankings 2018 para América Latina (<www.topuniversities. com>), de donde se eligió para cada país a diez universidades: cinco públicas y cinco privadas. Se hizo esto para evitar que el carácter público o privado de las instituciones introdujera un sesgo en los análisis, considerando que dicha característica influiría en las políticas de acceso a los servicios. Tomarlas de aquella clasificación sirvió como un criterio aproximadamente indicativo de que se trataba de las "mejores" universidades de cada país y que, consecuentemente, tendrían bibliotecas manejadas profesionalmente y con los más altos estándares de práctica bibliotecaria conocidos en sus contextos nacionales.

Acto seguido, se buscó en los sitios web de las universidades los reglamentos de servicios de sus bibliotecas centrales o sistemas de bibliotecas 
(disponibles en agosto de 2018); y de no haberlos, de al menos tres bibliotecas adscritas a dependencias académicas, o simplemente se descartó el caso por falta de información y se tomó a otra institución del posicionamiento. ${ }^{1}$ Para cada biblioteca o sistema se analizó:

1) Si contemplaba o no ofrecer servicios a usuarios externos; luego, si lo consideraba.

2) Cómo definía a estos usuarios (con qué atributos o derechos).

3) Si establecía o no requisitos especiales para su acceso al servicio básico de "lectura en sala" (o el mismo servicio con alguna otra denominación).

Se identificó así un extenso rango de situaciones, que fueron objeto de un subsecuente ordenamiento asociado a la construcción de una escala jerárquica con cinco categorías, a las que se atribuyó valores para representar los grados de apertura o restricción frente a los usuarios externos, de tal suerte que, a mayor puntaje, mayor sería el nivel de exclusión:

La información recopilada se ingresó a una hoja de cálculo de Excel, en la que se asignó a cada caso de observación un valor, según la escala, en atención a lo establecido en los diversos reglamentos, y se registró también la presencia o ausencia de requisitos específicos:

1) Tramitación de carnés para usuarios externos.

2) Entrega de fotografías.

3) Realización de pagos.

4) Presentación de cartas de respaldo institucional.

5) Presentación de otros documentos (recibos de servicios domiciliar certificaciones, etcétera).

6) Evaluación de la solicitud para su aprobación o rechazo.

7) Adscripción del solicitante a determinada categoría social o administrativa.

8) Atención en horarios diferenciados o restringidos.

Luego, se efectuó un cálculo sumatorio con los valores correspondientes a los grupos de bibliotecas de cada país, obteniéndose medias aritméticas que se atribuyeron a los casos nacionales para fines de comparación.

1 En el caso de Bolivia, se complementó la lista con el UniRank - Top Universities in Bolivia 2018 (<www.4icu.org/bo>), debido al reducido número de universidades bolivianas en QS. 
Escala de categorías para evaluar los niveles de apertura o restricción a la atención a usuarios externos en los servicios de bibliotecas universitarias

- Acceso irrestricto [1]: no se establecen requisitos para el acceso al servicio básico de consulta de materiales en los ambientes de la biblioteca, más allá de la presentación de alguna identificación y el seguimiento de normas de civilidad y cuidado de materiales e instalaciones.

- Acceso inmediato con restricciones menores [2]: se permite el acceso en el mismo día en que el usuario se acerca a solicitarlo, condicionado a la realización de algún procedimiento administrativo que la persona puede cumplir en ese mismo momento, incluyendo pagos, registros, llenado de formularios u otro trámite singular, menor y específico.

- Acceso restringido o diferido sujeto trámites burocráticos [3]: se permite el acceso a usuarios externos, siempre y cuando estos realicen previamente algún trámite complejo, que implica dos o más requisitos. Incluye la tramitación de carnés de biblioteca, que suele contemplar la presentación de formularios y solicitudes, la realización de pagos y la entrega de fotografías. Incluye la atención en horarios diferenciados y restringidos.

- Restricciones mayores para el acceso [4]: el acceso está condicionado a trámites complejos, sumados al requisito de que las personas tengan algún tipo de afiliación institucional o encajen en ciertos perfiles establecidos, o la solicitud de documentos distintos de la identificación oficial de ciudadanía y generados por otras instituciones (cartas de presentación o similares), y opcionalmente la evaluación de la solicitud, que puede ser aprobada o rechazada.

- Exclusión extrema o absoluta [5]: la biblioteca no contempla ofrecer servicios a usuarios externos por su sola condición de ciudadanía, o lo considera excepcionalmente en casos muy específicos, previa evaluación y con restricciones mayores que, además, requieren de la clasificación de la persona en determinada categoría social o administrativa, considerándose también la acreditación formal de su pertenencia a aquella categoría ("investigador", "tesista" o similares). 


\section{Resultados}

El Cuadro 1 presenta los resultados generales de la evaluación, con las siglas o abreviaturas de las setenta universidades representando a sus bibliotecas, más los valores asignados de acuerdo con la escala de acceso y restricción. En el Cuadro 1 identificamos tres grandes situaciones: en la primera, tenemos a las bibliotecas de Brasil, México y Argentina, donde los usuarios externos son ampliamente bienvenidos (con pocos casos fuera del patrón); en segundo lugar, vemos que en Colombia, Chile y Bolivia la situación es muy variada, con un buen número de instituciones que aceptan a los usuarios externos o les ponen pocas trabas, junto a otras que les colocan fuertes barreras, aunque en general las primeras dominan la escena. Y, finalmente, destaca en tercer lugar el singular caso de Perú, cuyas bibliotecas universitarias por lo regular han institucionalizado serios impedimentos al acceso de usuarios externos, cuando no lo prohíben casi por completo.

\begin{tabular}{|l|l|c|l|l|l|c|}
\hline \multicolumn{1}{|c|}{ Países } & Universidades & Escala & & \multicolumn{1}{|c|}{ Países } & Universidades & Escala \\
\hline BRASIL & UNICAMP & 1 & & CHILE & UCHILE & 1 \\
\hline U. públicas: & USP & 1 & $\begin{array}{l}\text { U. } \\
\text { públicas: }\end{array}$ & USCH & 4 \\
\hline & UFRJ & 1 & & & UValparaíso & 2 \\
\hline & UNESP & 1 & & & UBIO & 2 \\
\hline & UFMG & 1 & & & UTA & 1 \\
\hline & PUC-Rín & 1 & & U. privadas: & UCCL & 5 \\
\hline & PUCSP & 1 & & & Uconcepción & 2 \\
\hline & PUCRS & 1 & & & PUCV & 2 \\
\hline & Mackenzie & 1 & & & UDP & 5 \\
\hline & PUCPR & 2 & & & UTFSM & 3 \\
\hline MÉXIC0 & UNAM & 1 & & BOLIVIA & UMSA & 2 \\
\hline U. públicas: & IPN & 1 & & U. públicas: & UMSS & 3 \\
\hline & UAM & 1 & & & UTO & 2 \\
\hline & UAEM & 1 & & & UAGRM & 3 \\
\hline & UDG & 1 & & & UAB & 3 \\
\hline U. privadas: & TeC & 2 & & U. privadas: & UCB & 2 \\
\hline
\end{tabular}




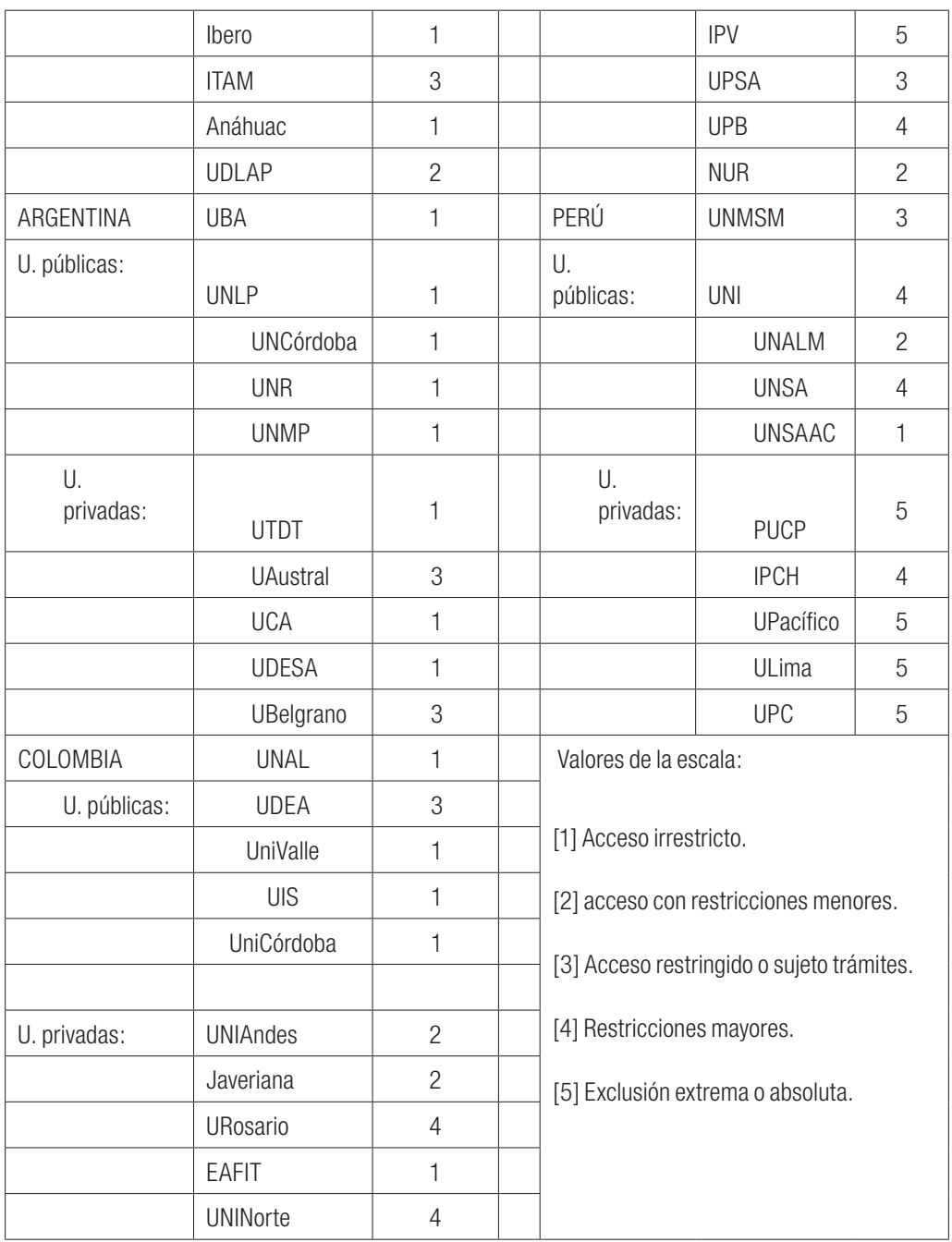

Cuadro 1. Accesibilidad y restricción al servicio de lectura en sala para usuarios externos en bibliotecas universitarias latinoamericanas.

Fuente: Elaboración propia, a partir de los reglamentos de setenta bibliotecas universitarias.

El Cuadro 2 recoge para cada país el cálculo de las medias aritméticas de los valores de la escala, lo que permite observar diferencias globales entre países. Pero, además, se observa aquí que las barreras y exclusiones son considerablemente más comunes en las instituciones privadas de casi todos los países. No obstante, también en esta categoría las diferencias son notables. 
De hecho, la distancia entre los extremos se amplifica más aún en el análisis separado de las bibliotecas del régimen privado, con las de Perú sobresaliendo por su estrecha cercanía (4.8) al mayor extremo de exclusión.

\begin{tabular}{|l|c|c|c|}
\hline \multicolumn{1}{|c|}{ País/región } & $\begin{array}{c}\text { Media } \\
\text { nacional } \\
\text { (escala) }\end{array}$ & $\begin{array}{c}\text { Universidades } \\
\text { públicas }\end{array}$ & $\begin{array}{c}\text { Universidades } \\
\text { privadas }\end{array}$ \\
\hline Brasil & 1.1 & 1 & 1.2 \\
\hline México & 1.3 & 1 & 1.8 \\
\hline Argentina & 1.4 & 1.4 & 1.4 \\
\hline Colombia & 2 & 1.4 & 2.6 \\
\hline Chile & 2.7 & 2 & 3.4 \\
\hline Bolivia & 2.8 & 2.6 & 3.2 \\
\hline Perú & 3.8 & 2.8 & 4.8 \\
\hline América Latina & 2.2 & 1.7 & 2.7 \\
\hline
\end{tabular}

Cuadro 2. Medias nacionales de los niveles de accesibilidad y restricción al servicio básico de lectura en sala para usuarios externos en bibliotecas universitarias.

* El valor 1 representa una apertura total y los valores más altos indican mayores niveles de restricción, con el 5 como máximo. Fuente: Elaboración propia, a partir de la Base de datos de los reglamentos de setenta bibliotecas universitarias.

El Cuadro 3 muestra cómo se manifiesta en Perú la pronunciada inclinación a establecer restricciones a los usuarios externos. Fuera del caso excepcional de la unsaac, las bibliotecas menos restrictivas (UNMSM y UNALM) solicitan dinero para permitir el acceso a sus servicios. Otras lo conceden previo cumplimiento de diversas condiciones, como estar afiliado a alguna institución que respalde la solicitud de atención (en todas las bibliotecas de universidades privadas), ser "investigadores", ser estudiantes universitarios de determinado nivel ("a partir del 8vo ciclo", PUCP), o ser "mayores de edad" (UNMSM).

En algunos escenarios, los usuarios externos deben tramitar carnés de biblioteca (que implican pagos, fotografías, solicitudes u otros documentos). La UNSA requiere una "carta de garantía" de "un docente o servidor administrativo titular" de esta universidad. También, en ocasiones, se exige que los solicitantes anuncien por anticipado sus temas de interés, mediante comunicaciones avaladas por una institución (UPC y UPacífico), pedidos que se evalúan para su aprobación o rechazo. 

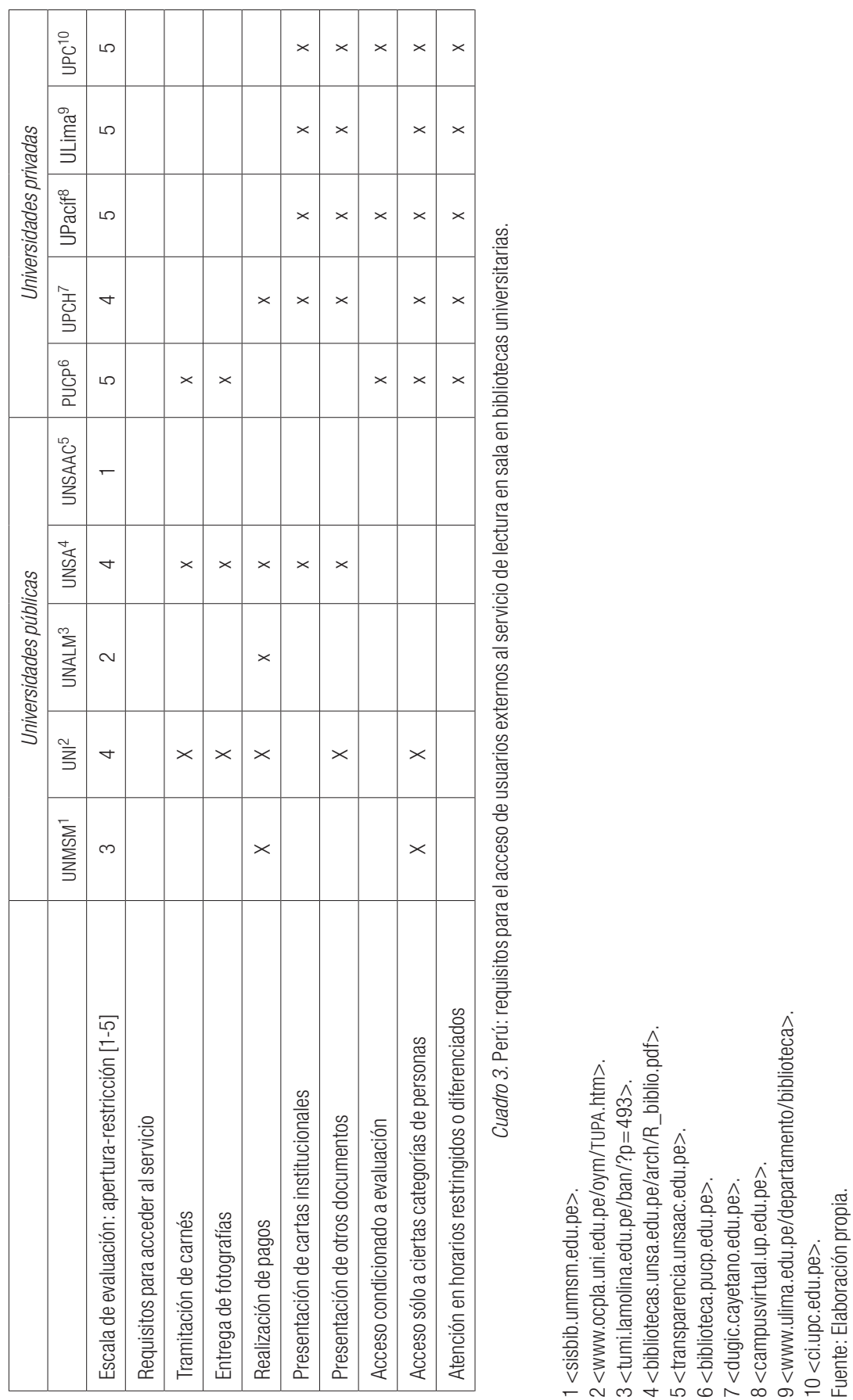


\begin{tabular}{|c|c|}
\hline Restricciones a usuarios externos & Apertura a usuarios externos \\
\hline $\begin{array}{l}\text { UNSA (Perú, pública): "Todo usuario deberá } \\
\text { presentar su carnet de Biblioteca [...]. Re- } \\
\text { quisitos: a) fotografía tamaño carné, llenar } \\
\text { un formulario... y el pago respectivo [...]. g) } \\
\text { En caso de que el interesado no pertenezca } \\
\text { a la Universidad, deberá presentar además } \\
\text { una carta de garantía en la que un docente } \\
\text { o servidor administrativo titular se responsa- } \\
\text { bilice por las obras que consulte la persona } \\
\text { garantizada". } \\
\text { PUCP (Perú, privada): "LECTURA EN SALA } \\
\text { PARA USUARIOS EXTERNOS. Requisitos: Estu- } \\
\text { diantes de pregrado o posgrado, profesores, } \\
\text { tesistas e investigadores.... Procedimiento: } \\
\text { Envía una solicitud a [...] directora del Sis- } \\
\text { tema de Bibliotecas, con copia a [...] coor- } \\
\text { dinadora de Acceso y préstamo. [...] Cada } \\
\text { caso será evaluado [...] CARNÉ DE LECTOR VI- } \\
\text { SITANTE - CLV: El Sistema... otorga en cada } \\
\text { semestre académico un determinado número } \\
\text { de carnés... a alumnos a partir del 8vo ciclo, } \\
\text { profesores o tesistas [...]. Presentar: Carta } \\
\text { de presentación en papel membretado de tu } \\
\text { universidad [...] según el modelo PUCP que } \\
\text { deberá estar sellada y firmada por el rector, } \\
\text { decano o director de estudios de su univer- } \\
\text { sidad [...] Fotocopia de tu DNI. Dos fotogra- } \\
\text { fías tamaño carné. Condiciones: 2. Los CLV } \\
\text { tendrán validez solo por un semestre [...] se } \\
\text { atenderá en los meses de abril y junio, y... } \\
\text { setiembre y noviembre [...] El número de car- } \\
\text { nés emitidos por semestre es limitado [...]". } \\
\text { uPC (Perú, privada): "Servicios para investi- } \\
\text { gadores de otras instituciones: los miercoles } \\
\text { (sic) de } 14.00 \text { a 20.00 horas. [...] Requisi- } \\
\text { tos: 1. Enviar previamente [...] una carta de } \\
\text { presentación de la institución a la que per- } \\
\text { tenece, en la que se indique el tema de su } \\
\text { fecha de visita [...]". }\end{array}$ & 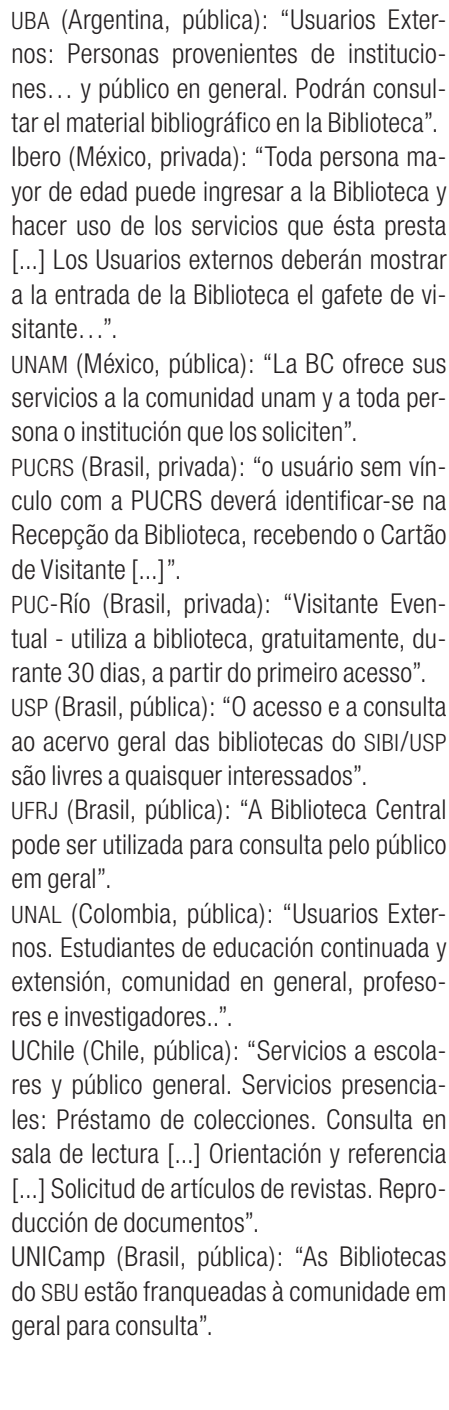 \\
\hline
\end{tabular}

Cuadro 4. Una mirada a los extremos.

Fuente: Elaboración propia, a partir de los reglamentos de servicios de las bibliotecas universitarias. 


\section{DisCUSIÓN Y CONCLUSIONES}

Este estudio de los reglamentos de setenta bibliotecas universitarias de siete países de América Latina revela que existen amplias diferencias en las posturas que éstas asumen frente al acceso de usuarios externos a sus servicios: en Brasil, México y Argentina, las bibliotecas universitarias suelen acoger a cualquier ciudadano; en Colombia, Chile y Bolivia se presentan variados niveles de apertura y restricción en distintas universidades, y en Perú, las bibliotecas por lo regular imponen a los usuarios externos múltiples requisitos para acceder a la atención, ubicándose en su mayoría próximas al mayor extremo de exclusión definido en la escala utilizada para la evaluación.

Por otra parte, las restricciones son más comunes en las instituciones privadas, cosa que se ha identificado en investigaciones previas (Weare y Stevenson, 2012) y que sería esperable, aunque no lo es tanto si consideramos que, en conjunto, las bibliotecas de universidades privadas latinoamericanas son en promedio menos restrictivas que las públicas de Perú.

Éste es el primer estudio que analiza, describe y compara la situación del acceso por parte de usuarios externos a los servicios de bibliotecas universitarias a nivel de América Latina. En tal sentido, constituye una contribución original al conocimiento sobre este tema. Los hallazgos indican que en una parte importante de esta región dichas bibliotecas operan bajo una filosofía de proyección social universitaria que tiene una larga tradición en el mundo bibliotecario. Por ejemplo, ya en 1775 la biblioteca de la Universidad de Salamanca según el reglamento de ese año ofrecía sus servicios a "los naturales, i personas aficionadas de el pueblo" (Becedas, 1995: 48). Asimismo, Waggoner (1964) señala que, en Estados Unidos, desde inicios del siglo xx, los bibliotecarios de muchas universidades han abogado por la apertura de sus servicios a la comunidad. En este país, un estudio de 1965 encontró que, en ese año, el 94 por ciento de 783 bibliotecas académicas ofrecía sus servicios a usuarios externos (Josey, 1969), mientras que otro estudio de 2001, esta vez con 527 bibliotecas académicas, halló que todas atendían a usuarios externos, siendo el acceso "irrestricto" en el 89 por ciento de los casos (96 por ciento en instituciones públicas) (Courtney, 2003). Resulta entonces que, a escala latinoamericana, al menos en Brasil, México y Argentina, se ha adoptado esta doctrina de apertura y proyección a la sociedad que está ya bien establecida en los países más desarrollados (Atkinson, 2018; Russell et al., 1992) y que se asume incluso como una obligación "ética" de los bibliotecarios (Martin, 1990). 
En contraste, se da en Perú una situación diametralmente opuesta, con restricciones múltiples para el acceso de usuarios externos, o su completa exclusión en no pocos casos (como cuando el término "usuario externo" se refiere únicamente a ciertos tipos de personas, mas no a cualquier ciudadano del país). Este escenario se asemeja ligeramente a lo que Herrera y Pérez (2009) han encontrado en España, aunque anotando que allí los condicionamientos son "asequibles en la mayoría de los casos".

Es preciso reconocer algunas limitaciones del estudio. Su metodología no está exenta de sesgos y errores en los procedimientos de análisis o en las propias fuentes de información. Como en otros trabajos basados en reglamentos de bibliotecas (Herrera, 2001; 2003), también en éste los documentos fueron de calidad desigual, algunos con ambigüedades o hasta contradicciones, lo que exigió esfuerzos de interpretación que, en ciertos casos, pudieron haberse apartado de las intenciones originales de sus autores. Asimismo, los reglamentos no necesariamente reflejan las labores reales de las bibliotecas. Por ejemplo, algunos muy restrictivos serían empleados de manera flexible en los servicios. O quizás ocurriría también que normas favorables al libre acceso de usuarios externos no sean cumplidas por algunos operadores especialmente celosos e inclinados a establecer barreras informales a la atención. Sea como fuere, el método ofrece ventajas nada desdeñables, como la de facilitar la comparación de numerosos casos y la identificación de diferencias a escala nacional.

Este último punto pone de relieve los extremos de la comparación. No cabría sacar aquí conclusiones sobre el porqué de la singularidad del caso peruano, debido al carácter exploratorio de este estudio, pero se puede adelantar al menos algunas hipótesis. Para empezar, no parece que estemos ante un problema de formación bibliotecaria. En diversos campos específicos (como el desarrollo de colecciones y bases de datos, o el manejo gerencial), los bibliotecarios peruanos exhiben desempeños comparables a los de sus colegas de Brasil, México o Argentina, y no son ajenos a la participación en foros internacionales donde se discuten las novedades de su disciplina. Además, nos referimos a una élite profesional ubicada en las universidades más importantes y prestigiosas del país, incluyendo a las que ofrecen formación en bibliotecología (UNMSM y PUCP). Luego, la comparación entre países lleva a relativizar las justificaciones que desde las propias bibliotecas se alegarían para imponer barreras de acceso (como costos, recursos, personal, espacio, etc.), pues a primera vista nada lleva a pensar, por ejemplo, que las bibliotecas peruanas tengan en general más limitaciones que las bolivianas.

Lo observado en Perú se explicaría por las condiciones de la ciudadanía y la democracia en este país. Las ciencias sociales peruanas han insistido en señalar que existen aquí enormes carencias en la aceptación de valores 
cívicos y democráticos, como la igualdad de las personas y los derechos ciudadanos, sosteniendo que predomina, en cambio, la valoración de los individuos de acuerdo a su estatus social, su "educación", su etnicidad, su procedencia geográfica y hasta su apariencia física, tanto en las interacciones cotidianas (Santos, 2002), como en espacios institucionales. Y aun cuando estos problemas se dan en todo el espacio latinoamericano, diversos análisis coinciden en colocar a Perú entre los países latinoamericanos donde menor fuerza y relevancia tienen las instituciones democráticas y los valores ciudadanos (Carrión et al., 2012).

\section{CONCLUSiones}

La comparación de las políticas de acceso a los servicios en las bibliotecas universitarias latinoamericanas revela que entre sí existen enormes diferencias en sus formas de relacionarse con los ciudadanos de sus países. El panorama mostrado invita a nuevas indagaciones para esclarecer lo que estaría detrás de esas diferencias, prestando atención tanto a las políticas y prácticas bibliotecarias, como a las filosofías que las definen, no sólo en materia de doctrina bibliotecaria, sino también en lo que concierne a criterios de proyección social, democracia, ciudadanía e igualdad de las personas.

$\mathrm{Al}$ respecto, en el caso extremo de Perú, no parece casual que los reglamentos de sus bibliotecas universitarias señalen en su mayoría y tan enfáticamente que los usuarios externos deben pertenecer a determinadas categorías sociales (indicadoras de su estatus) para merecer atención, o que necesiten acreditar sus antecedentes educativos y de procedencia institucional, dejándose relegada casi siempre su sola condición de ciudadanía como un criterio para aceptarlos en los servicios. En estos aspectos, las actitudes de los bibliotecarios, reflejadas en sus reglamentos, son en realidad muy similares a las que comúnmente gobiernan numerosos ámbitos institucionales en que se mueven los peruanos (en los servicios de salud, la administración de justicia, etcétera). Desde esta perspectiva, tales prácticas restrictivas no serían más que la reproducción, en el ámbito específico de la biblioteca universitaria, de los esquemas sociopolíticos y culturales que típicamente regulan las relaciones sociales entre las personas de este país.

Otra posible explicación estaría en las diferencias en los roles desempeñados por élites políticas e ilustradas en los arreglos institucionales y normativos de los países, en vista de que justamente Brasil, México y Argentina se han caracterizado por tener élites intelectuales y modernizantes con gran peso histórico y presencia política, mientras que en Perú los espacios de poder 
han acogido muy poco la participación y las propuestas democratizadoras de los intelectuales más progresistas.

Finalmente, no menos importantes son los impactos sociales derivados de los dos esquemas de práctica bibliotecaria identificados: por un lado, la proyección social universitaria a través de las bibliotecas beneficia a las universidades que la practican, fortaleciendo sus relaciones con las sociedades de sus entornos (O'Kelly, 2017; Torres, 2005) y enriqueciendo su imagen pública, ambas cosas a un costo muy bajo (Courtney, 2001; 2003; Weare y Stevenson, 2012), sin contar los beneficios que esto significa para la comunidad y su desarrollo; y por el otro, la exclusión de los usuarios externos y las negativas experiencias de ellos por su segregación, lógicamente generan justificadas percepciones masivas de desagrado, dirigidas no sólo a las bibliotecas y los bibliotecarios, sino también a las universidades implicadas, sin beneficios evidentes para estos actores.

\section{ReFERENCIAS}

Atkinson, Jeremy. 2018. "Collaboration and academic libraries: an overview and literature review", en Collaboration and the academic library: internal and external, local and regional, national and international. Cardiff, Wales: Chandos, pp. , 11-33.

Becedas, Margarita. 1995. "Primeros reglamentos en la biblioteca universitaria de Salamanca: 1775-1776”, en Sonsoles Angulo, coord., De libros y bibliotecas: homenaje a Rocío Caracuel. Sevilla: Universidad de Sevilla, pp. 37-48.

Carrión, Julio, P. Zárate y M. Seligson. 2012. Cultura política de la democracia en Perú, 2012: hacia la igualdad de oportunidades. Lima: USAID.

Courtney, N. 2001. "Barbarians at the gates: A half-century of unaffiliated users in academic libraries". Journal of Academic Librarianship 27 (6): 473-480, en $<$ https://doi.org/10.1016/S0099-1333(01)00260-9>.

Courtney, N. 2003. "Unaffiliated users' access to academic libraries: A survey”. Journal of Academic Librarianship 29 (1): 3-7, en <https://doi.org/10.1016/S00991333(02)00387-7>.

Herrera, José. 2003. "Estudio del préstamo general y de los préstamos especiales en las bibliotecas universitarias españolas a través de sus normativas y reglamentos". Revista Española de Documentación Científica 26 (3): 306-340, en <https://doi. org/10.3989/redc.2003.v26.i3.140>.

Herrera, José. 2001. "Contenido y estructura de los reglamentos de las bibliotecas universitarias de Andalucía”. Boletín de la Asociación Andaluza de Bibliotecarios 62: 7-30, en <http://eprints.rclis.org/5964/>.

Herrera, José y Margarita Pérez. 2009. "La función social en las bibliotecas universitarias españolas: planes, usuarios y actividades”. Zaragoza: ponencia presentada en Interinformación: XI Jornadas Españolas de Documentación, 20-22 de mayo. 
Josey, E. J. 1969. “Community use of academic libraries”. Library Trends 18 (1): 66-74, en <http://hdl.handle.net/2142/6478>.

Mainka, Agnes, S. Hartmann, L. Orszullok, I. Peters, A. Stallmann y W. Stock. 2013. "Public libraries in the Knowledge Society: core services of libraries in informational world cities”. Libri 63 (4): 295-319, <https://doi.org/10.1515/libri-2013-0024>.

Martin, R. 1990. “The paradox of public service: where do we draw the line?”. College EResearch Libraries 51: 20-26, en <https://doi.org/10.5860/crl_51_01_20>.

Materska, Katarzyna. 2004. "Librarians in the knowledge age". New Library World 105 (3-4): 142-148, en <https://doi.org/10.1108/03074800410526776>.

O'Kelly, Mary. 2017. "Community success: rethinking public services in academic libraries”. International Information E Library Review 49 (3): 225-229, en <https:// doi.org/10.1080/10572317.2017.1353372>.

Russell, R., C. Robison, J. Prather y C. Carlson. 1992. "External user access to academic libraries in urban/metropolitan areas", en G. McCabe, ed., Academic libraries in urban and metropolitan areas: A management bandbook. Westport: Greenwood Press, pp. 27-32.

Santos, Martín. 2002. "La 'cuestión racial': un ajuste de cuentas en tiempos de globalización y postmodernidad”. Debates en Sociología 27: 133-171, en <http://revistas. pucp.edu.pe/index.php/debatesensociologia/article/view/7052>.

Stilwell, Christine. 2018. "Information as currency, democracy, and public libraries". Library Management 39 (5): 295-306, en <https://doi.org/10.1108/LM-08-2017-0078>.

Torres, Marta. 2005. "La función social de las bibliotecas universitarias". Boletín de la Asociación Andaluza de Bibliotecarios 80: 43-70, en <https://dialnet.unirioja. es/descarga/articulo/2173486.pdf>.

Waggoner Jr., John P. 1964. "The role of the private university library”. North Carolina Libraries 22:55-57.

Weare Jr., W. y M. Stevenson. 2012. "Circulation policies for external users: A comparative study of public urban research institutions". Journal of Access Services 9 (3): 111-133, en <https://doi.org/10.1080/15367967.2012.684563>.

Para citar este texto:

Nureña, César R. 2019. "Bibliotecas universitarias y proyección social: diferencias y extremos en América Latina”. Investigación Bibliotecológica: archivonomía, bibliotecología e información 33 (80): 117-132. http://dx.doi.org/10.22201/iibi.24488321xe.2019.80.58009 